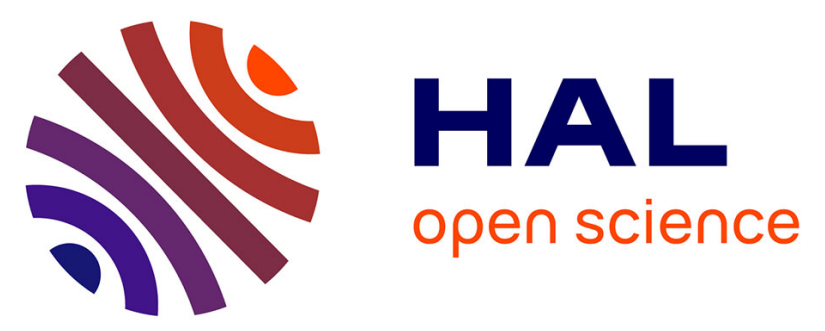

\title{
L'interaction réflexivité-intelligence motrice dans les arts martiaux. Le cas de l'initiation rituelle penca à Banten (Indonésie)
}

Gabriel Facal

\section{- To cite this version:}

Gabriel Facal. L'interaction réflexivité-intelligence motrice dans les arts martiaux. Le cas de l'initiation rituelle penca à Banten (Indonésie). STAPS : Revue internationale des sciences du sport et de l'éducation physique, 2010, 89, 10.3917/sta.089.0011 . hal-03046642

\section{HAL Id: hal-03046642 \\ https://hal.science/hal-03046642}

Submitted on 4 Jan 2021

HAL is a multi-disciplinary open access archive for the deposit and dissemination of scientific research documents, whether they are published or not. The documents may come from teaching and research institutions in France or abroad, or from public or private research centers.
L'archive ouverte pluridisciplinaire HAL, est destinée au dépôt et à la diffusion de documents scientifiques de niveau recherche, publiés ou non, émanant des établissements d'enseignement et de recherche français ou étrangers, des laboratoires publics ou privés. 


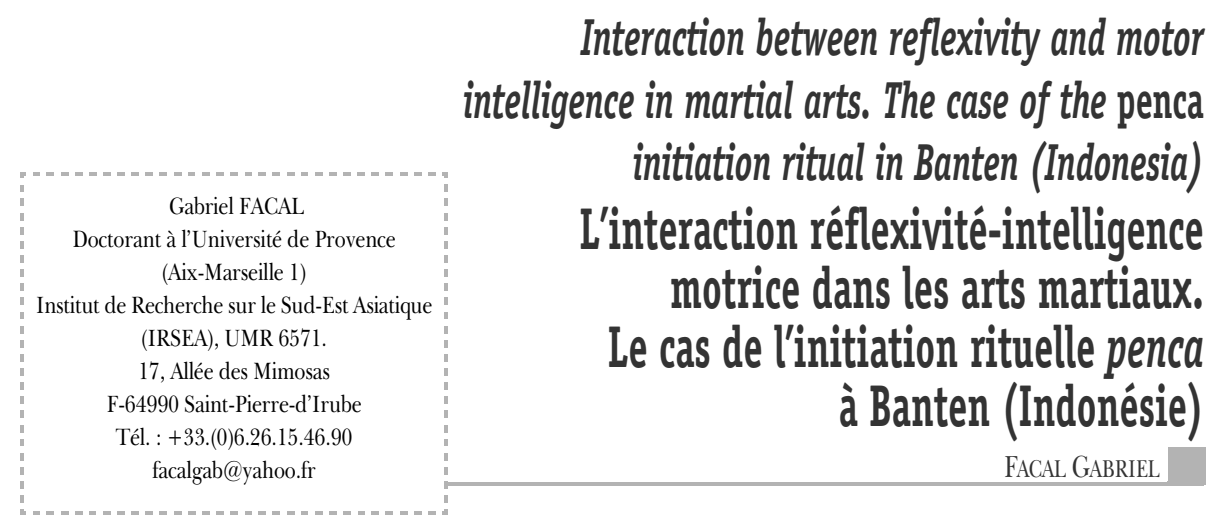

RÉsumÉ : L'étude porte sur la relation qu'entretiennent réflexivité et intelligence motrice dans le processus d'apprentissage et la pratique des techniques martiales de l'initiation rituelle penca, telle qu'on la trouve dans le village de Rancalame (Banten, Indonésie). Dans ce contexte, l'analyse de la progression de l'apprentissage semble attester que la réflexivité est sollicitée aux niveaux élémentaires de l'initiation mais que progressivement elle cède la place à des modes de pratique qui favorisent l'intelligence motrice. Les pratiquants de niveau ultime valorisent fortement la créativité, l'improvisation et la personnalisation des mouvements. En comparant les divers niveaux de pratique, il semble que ce phénomène soit intrinsèquement lié au processus de socialisation des pratiquants. En effet, depuis les enfants jusqu'aux experts, ceux-ci se trouvent impliqués de façon croissante dans un ordre social statutaire hiérarchisé. Après avoir décrit cette initiation rituelle, je montrerai comment elle s'accorde avec des ensembles techniques exogènes qui valorisent la réflexivité et la rationalisation des techniques. Le texte s'inscrit à la suite de celui de Billeter (1984), qui met en évidence qu'en certains contextes, pensée et acte ne sont pas dissociés, et il prolonge l'analyse de Grave (2001, 2007) qui examine l'interaction entre technique et valeur.

MOTS CLÉS : réflexivité, intelligence motrice, techniques, valeurs, initiation rituelle.

ABSTRACT: This study concerns the relation between reflexivity and motor intelligence in the learning process of martial art techniques in the penca initiation ritual, as found in the village of Rancalame (West Java, Indonesia). In this context, the analysis of the learning progress seems to attest that reflexivity is solicited at basic levels of the initiation, but that progressively it lose place to practice modes which are favored by motor intelligence. The practitioners at an advanced level strongly value creativity, improvisation and personalization of movements. In comparing the different levels of practice, it seems that this phenomenon is intrinsically bound to the practitioners' socialization process. In effect, from children to experts, at each stage they are increasingly implicated in a hierarchical order of social status. After describing the initiation ritual, I will show how it incorporates exogenic group techniques which value their rationalization and reflexivity. The analysis is based on the work of Billeter (1984), who shows that in certain contexts thinking and acting are not dissociated, and further expands on de Graves' studies (2001, 2007), which examine the interaction between technique and value.

KEY WORDS: reflexivity, motor intelligence, techniques, values, initiation ritual. 


\section{Zusammenfassung: Die Interaktion zwischen Reflexion und motorischer Intelligenz in den Kampfsportarten. Das Beispiel der rituellen penca in Westjava (Indonesien)}

Bei der Untersuchung geht es um den Zusammenhang zwischen Reflexion und motorischer Intelligenz im Lernprozess der Kampftechniken des Initiationsritus penca, wie man ihn in dem Dorf Rancalame (Westjava, Indonesien) vorfindet. In diesem Kontext scheint die Analyse des Lernfortschritts zu zeigen, dass die Reflexion auf den elementaren Ebenen der Initiation gefordert wird, aber nach und nach Ausübungsmodalitäten Platz macht, bei denen die motorische Intelligenz überwiegt. Bei den Akteuren des höchsten Niveaus werden die Kreativität, die Improvisierung und die Personalisierung der Bewegung besonders wichtig. Vergleicht man die unterschiedlichen Leistungsniveaus, so scheint es, dass dieses Phänomen intrinsisch an den Prozess der Sozialisation der Akteure gebunden ist. In der Tat, von den Kindern bis zu den Experten, finden sich diese zunehmend in einer hierarchisierten Ordnung des Sozialstatus wieder. Nach der Beschreibung dieser rituellen Initiation werden wir zeigen, wie sie sich einer Gesamtheit von exogenen Techniken anpasst, die Reflexion und Rationalisierung der Techniken in den Vordergrund hebt. Der Text basiert auf den Arbeiten von Billeter (1984), der zeigt, dass in gewissen Kontexten Denken und Handeln nicht getrennt sind, und vertieft die Analyse von de Grave (2001, 2007), der die Interaktion zwischen Technik und Werten untersucht.

SCHLAGWÖRTER : reflexion, motorische Intelligenz, Techniken, Werte, rituelle Initiation.

\section{Resumen : La interacción reflexividad-inteligencia motriz en las artes marciales. El caso de la iniciación ritual penca en Java Oeste (Indonesia)}

El estudio se apoya en la relación que mantienen reflexividad e inteligencia motriz en los procesos de aprendizaje y la práctica de las técnicas marciales de la iniciación ritual penca, tal y como se encuentran en el pueblo de Rancalame (Java Oeste, Indonesia). En ese contexto, el análisis de la progresión del aprendizaje parece atestigüar que la reflexividad es solicitada en los niveles elementales de la iniciación pero que progresivamente cede espacio a modos de práctica que favorecen la inteligencia motriz. Los practicantes de último nivel valoran fuertemente la creatividad, la improvisación y la personalización de los movimientos. Comparando los diversos niveles de práctica, parece que este fenómeno es intrínsicamente ligado a los procesos de socialización de los practicantes. En efecto, desde los niños hasta a los expertos, se encuentran implicados de manera creciente en un orden social estatutario jerarquizado. Después de haber descrito esta iniciación ritual, se mostrará como concuerdan con conjuntos técnicos exógenos que valoran la reflexividad y la racionalización de las técnicas. El texto se inscribe a continuación de aquel de Billeter (1984) que muestra que en ciertos contextos, pensamientos y actos no se disocian, y se prolonga el análisis de de Grave $(2001,2007)$ que examina la interacción entre la técnica y el valor.

Palabras Claves : reflexividad, inteligencia motriz, técnicas, valores, iniciación ritual.

\section{RIAssunto : L'interazione riflessività-intelligenza motoria nelle arti marziali. Il caso dell'iniziazione rituale penca a Java Ovest (Indonesia)}

Lo studio è basato sulla relazione che mantiene riflessività e intelligenza motoria nel processo d'apprendimento e la pratica delle tecniche marziali dell'iniziazione rituale penca, come si trova nel villaggio di Rancalame (Java Ovest, Indonesia). In questo contesto, l'analisi della progressione dell'apprendimento sembra attestare che la riflessività sollecitati i livelli elementari dell'iniziazione ma che progressivamente cede il posto a modi di pratica che favoriscono l'intelligenza motoria. I praticanti del livello ultimo valorizzano fortemente la creatività, l'improvvisazione e la personalizzazione dei movimenti. Comparando i diversi livelli di pratica, sembra che questo fenomeno sia intrinsecamente legato al processo di socializzazione dei 
praticanti. In effetti, dai bambini fino agli esperti, questi ultimi si trovano implicati in maniera crescente in un ordine statutario gerarchizzato. Dopo aver descritto quest'iniziazione rituale, si mostrerà come essa si accorda come degli insiemi tecnici esogeni che valorizzano la riflessività e la razionalizzazione delle tecniche. Il testo si inscrive nelle indagini di Billeter (1984) che mostra che in certi contesti, pensiero e atto non sono dissociati, e io prolungo l'analisi di de Grave $(2001,2007)$ che esamina l'interazione tra la tecnica e il valore.

PAROLE CHIAVE : iniziazione rituale, intelligenza motoria, riflessività, tecniche.

\section{INTRODUCTION}

Dans le village de Rancalame (Banten, Indonésie), l'apprentissage des techniques martiales s'inscrit dans l'initiation rituelle penca, qui comprend la transmission de savoirs et de savoir-faire liés à la religion musulmane, à la relation aux esprits des ancêtres et d'animaux tutélaires du lieu. Dans ce processus d'apprentissage, l'introduction de la réflexivité dans l'exécution des mouvements semble peu marquée, tandis que l'intelligence motrice est éduquée dès les niveaux élémentaires de la pratique. L'analyse de la progression des initiés semble attester que l'intelligence motrice prend rapidement le relais de la réflexivité. Conjointement, ceux-ci sont progressivement investis de responsabilités ainsi que d'un rôle dans les réseaux d'échange et de relations intra- et extra-villageois.

Dans le village, le dispositif de transmission est qualifié de traditionnel (asli) et le corpus technique qui s'y rattache forme un système. Celui-ci est caractérisé par une grande capacité d'intégration de techniques exogènes au travers desquelles transparaît une volonté de rationalisation et de simplification des mouvements. L'interaction entre un système technique - dont les propriétés valorisent l'intelligence motrice et des pratiques de type réflexif semble contradictoire.

Ce phénomène d'intégration de techniques fondées sur des dynamiques, en apparence opposées à celles qui animent le système de pratique, sera analysé à la lumière de la relation qu'entretiennent les techniques avec un ensemble de valeurs ordonnées. Je montrerai que la nature du système technique autorise une grande variété de déclinaisons à partir d'un même mouvement, et que la créativité et l'innovation sont au cœur de la dynamique de cette pratique. Cela permettra de penser la capacité d'un système technique à se modifier tout en conservant ses propriétés fondamentales. Ainsi, c'est le rapport du pratiquant à lui-même - à travers la réflexivité - et à l'altérité - l'intelligence motrice attestant la socialisation du sujet - qui se trouve questionné.

\section{L'ORGANISATION SOCIALE DU VILLAGE DE RANCALAME}

À Rancalame, les entraînements aux techniques martiales, comme la plupart des activités qui rassemblent l'ensemble du village, ont lieu dans la maison du maitre ou sur son domaine. Les pratiquants dorment régulièrement dans la pièce commune de sa maison ou sur les bancs de bambou qui entourent celle-ci. Son épouse et ses filles cuisinent quotidiennement pour une dizaine de personnes, et en contrepartie ces hôtes aident le maître aux travaux des rizières, à transporter les sacs de riz par camion jusqu'à la ville de Serang, ou à entretenir la maison. Le maitre qualifie ses disciples d'« enfants » (anak buah), tandis que ceux-ci s'adressent à lui comme à un "père" $(a b a h)$. De même, son épouse est appelée «mère » $(\mathrm{ma})$ par les pratiquants. Ceux-ci utilisent entre eux des termes marquant la relation classificatoire " aîné-cadet ". Elle peut être cogénérationnelle comme dans " petit frère ", "petite sœur ", "grand frère", " grande sœur », ou intergénérationnelle comme dans « oncle », « neveu », « tante », « nièce ». Les 
assistants du maître l'aident dans les travaux quotidiens et participent à la transmission du penca dans le village. Ils le suppléent aussi au niveau de l'organisation et du dispositif relationnel de l'école et du village avec les autorités locales, régionales et nationales. Le village compte environ deux cent cinquante habitants, dont beaucoup pratiquent ou ont pratiqué le penca avec le maitre. Celui-ci est connu dans les villages voisins, d'où les jeunes gens comme les anciens se rendent régulièrement chez lui pour étudier le penca, demander des conseils, obtenir des formules (jampe) et des amulettes (jimat) de protection ou suivre des soins. Les enfants du maître et la famille élargie jouent un rôle important dans la transmission des techniques de penca. Les enfants du maître ont en général un mode de résidence matrilocal mais ils vivent tous à proximité de sa maison et travaillent sur ses terres. Par contre, le fils cadet du maître doit consacrer ses années de jeunesse à éprouver sa formation à l'extérieur du village. Il vit en ville, travaille à l'usine tout en enseignant le penca aux membres d'une branche de l'école de son père. Il y est l'officiant cérémoniel et il initie les nouveaux pratiquants.

\section{LES ENTRAÎNEMENTS AU PENCA À RANCALAME}

Les entraînements au penca peuvent avoir lieu jusqu'à cinq soirs par semaine, la pratique étant proscrite le vendredi et le dimanche soir. Les heures de pratique ne sont pas formelles mais elles ont pour obligation de ne débuter qu'après la prière du soir (sholat Isya) à 19 heures et elles s'attardent parfois jusqu'à la prière du début du jour (sholat Subuh) à 5 heures le matin. Les pratiquants viennent de Rancalame ou des villages des alentours. Lorsqu'il pleut, ils s'entraînent dans la maison du maitre, autrement ils se rendent sur un terrain de terre battue (buruan) devant celle-ci. Parfois, le joueur de trompette (tarumpet) vient du village voisin, et on peut aussi amener l'orchestre (kendang) de gongs et tambours depuis la maison du maittre jusqu'au terrain d'entraînement.

Ce sont d'abord les plus jeunes qui s'entraînent aux danses et aux techniques formalisées à deux. En général, ils débutent la pratique entre eux et parfois un élève de niveau supérieur vient rectifier certains aspects techniques. Vers 22 heures, le second groupe de pratique relaie celui des enfants. Il est constitué d'élèves âgés de douze ans et plus, le dernier groupe étant constitué d'hommes mariés et de ceux en âge de le devenir. Les adolescents s'entrainent aux techniques d'attaques et de parades pratiquées à deux (kelid), tandis que les adultes peuvent accéder aux formes d'un niveau ultime de techniques au sol, les «jeux en bas » (ulin handap). Chez les premiers, il n'y a que rarement intervention des aînés et celles-ci se bornent souvent à une démonstration de certains mouvements qu'il faudra reproduire avec « l'intention » (niat) qu'il convient. En revanche, lorsque les adultes s'entraînent, le maitre sort de la maison et s'assied sur un banc à côté de l'aire d'entraînement. Il s'exprime parfois oralement pour donner des indications sur la façon d'exécuter les techniques de penca. Les filles et les femmes pratiquent le penca en commun avec les garçons et les hommes. Elles ont accès aux formes dansées et aux enchaînements de mouvements codifiés que l'on pratique seul (jurus) ou à plusieurs (jurus masal), mais elles n'ont pas le droit d'apprendre les jeux de parades et de contres avec les bras ou les formes de jeu au sol.

\section{LES TECHNIQUES DE PENCA}

\subsection{Les techniques fondamentales}

D'un point de vue technique, la structure d'enseignement est constituée d'attaques et de parades pratiquées à deux (kelid), de danses (igelan) et de techniques de combat au sol (ulin handap). Toutes ces pratiques sont qualifiées de jeux (ulinan) : on dit jouer aux parades, jouer sur l'estrade de démonstration, jouer au sol. Les démonstrations sont souvent accompagnées 
de pratiques d'invulnérabilité qui sont aussi qualifiées de jeux et qui sont enseignées de mâ̂tre à élève, de façon annexe aux entraînements en commun. Dans le répertoire du vocabulaire technique sont récurrents les termes : haut, bas, intérieur, extérieur, ascension, descente.

C'est ce qui apparaît dans le testament du maître fondateur de l'école où sont référencées toutes les techniques élémentaires. Celui-ci est énoncé par son fils aîné, le maître actuel de penca, lors du rituel pertalekan, au cours duquel l'initié doit faire des vœux et promesses. Selon ce texte, les techniques de penca trouvent leur origine et leur sens dans les mouvements de la prière musulmane. Par exemple, à partir du premier mouvement de la prière, le maitre fondateur a crée une danse qui est exécutée par le pratiquant pour appeler un esprit de tigre à le posséder. D'autres techniques avec ou sans armes, sont explicitement liées aux mouvements exécutés quotidiennement, comme ceux qui ont trait à l'élevage ou à la riziculture : bêcher, puiser de l'eau dans un puits, porter un sac de riz, tirer des fagots de bambou, ferrer les sabots d'un cheval, etc. Dans cette perspective, le travail quotidien est considéré comme faisant partie intégrante de l'entraînement du corps des pratiquants ainsi que de la formation aux techniques du penca.

Dans celles-ci, l'aspect relationnel est très valorisé. Le contrôle des émotions, les règles de comportement, comme celle de la préséance des aînés sur les cadets, celle de la dévotion des enfants envers leur parents, les règles d'obligation entre époux, et la relation aux ancêtres et aux esprits tutélaires sont au fondement des techniques de penca qui réclament maitrise du mouvement, équilibre, précision, gestion des distances interpersonnelles et de la relation à l'environnement physique. Par rapport à cela, le ressenti (rarasaan) est valorisé comme un indicateur de ce vers quoi il faut tendre dans la pratique. Le mouvement doit être ressenti comme bon, presque au sens culinaire : goûteux (raos).

\subsection{Les techniques exogènes et la spécialisation des initiés}

Chaque assistant du maître peut être récipiendaire d'une spécialisation dans laquelle il est incité à poursuivre sa formation. Par exemple, s'il possède des qualités particulières pour le maniement d'une arme, un pratiquant pourra être encouragé à étudier des techniques d'arme avec d'autres maitres, tout en poursuivant sa formation globale avec le maître de Rancalame. Celui-ci examinera le nouveau contenu technique acquis et pourra éventuellement l'intégrer au système technique d'ensemble de l'école. Il chargera ensuite son assistant de transmettre ses connaissances particulières aux autres initiés qui souhaitent approfondir leurs compétences dans ce domaine. Ainsi, la structure technique du système de penca est sans cesse étoffée. Cependant, les techniques intégrées à l'enseignement général le sont selon une perspective propre, où ce sont les techniques fondamentales - il s'agit des jeux que j’ai mentionné plus haut: les kelid, les igelan et les ulin handap qui forment le noyau dur et stable, très valorisé, et où c'est à partir de celui-ci et de façon subordonnée que les techniques nouvelles et extérieures s'ordonnent hiérarchiquement dans le système technique d'ensemble. De plus, ces techniques nouvellement intégrées sont souvent sujettes à modification dans la mesure où elles doivent s'adapter à l'ensemble des techniques fondamentales du système de penca. En revanche, les techniques fondamentales sont soumises à une moins grande porosité, elles sont moins pénétrées par les influences exogènes, qui ne les imprègnent qu'au cours d'un processus de long terme.

\section{LE PROCESSUS DE TRANSMISSION}

\subsection{Les étapes de l'apprentissage}

Après le rituel pertalekan, les assistants du maître transmettent à l'initié des «contenus " (eusi) : les techniques kelid et des enchaînements 
de mouvements esthétiques, dont on dit qu'ils sont la "fleur" (bungah ou kembang) du penca. Lors d'un rituel qui marque le passage de niveau, le maitre fait descendre (turun) l'esprit dans le corps du pratiquant. Cet adorcisme (kasurupan) permet à celui-ci de découvrir les techniques martiales que l'on nomme «fruit » (buah). La possession permet aussi d'apprendre les techniques ulin handap qui sont le prolongement des techniques kelid.

Le processus d'apprentissage se présente comme un cheminement vertical. Enfant, l'initié s'entraîne aux kelid en position assise. Plus tard, il apprend à se lever et à se déplacer, puis un autre rituel lui permet d'apprendre les ulin handap, techniques de niveau ultime, considérées comme "l'ascension au sommet » (naek ka puncak).

Comme on l'a vu, ce sont les rituels qui marquent l'accession aux différents niveaux de pratique.

\subsection{Le rituel keceran}

Les rituels sont intercomplémentaires et permettent au pratiquant d'accéder à certaines connaissances ésotériques. Il existe des rituels d'initiation, de passage de niveau, d'invulnérabilité et de mise au point d'huiles rituelles. Outre ceux-ci, le keceran est une cérémonie rituelle qui touche la communauté de penca en son entier. À cette occasion, le frère cadet du maitre passe dans l'assemblée et, à l'aide d'un couteau autour duquel est enroulée une feuille de bétel, il met des gouttes d'une solution aqueuse dans les yeux de chaque pratiquant. Cette opération leur permet d'entrer en relation avec les esprits ancestraux et les esprits de tigres. Lorsque tout le monde a reçu cette solution, un repas et une boisson à base de fruits, préparés par les femmes, sont consommés en commun. Le keceran est l'occasion de pratiquer les exorcismes, les transes, les debus, et de donner ainsi la parole aux esprits par l'intermé- diaire des pratiquants. À la fin du rituel, chacun se voit attribuer une part égale de nourriture.

Ont lieu des démonstrations où l'on danse le penca et où l'on pratique des adorcismes. Les possessions mettent souvent en scène la lutte d'un villageois - pas nécessairement initié au penca - contre un élément ou un événement qui a marqué la communauté pendant la période rituelle. Un litige, une querelle avec un village voisin, la venue d'un étranger, une union importante entre deux familles, pourront être représentés par un objet porté sur l'estrade de démonstration ou au travers d'un combat entre deux pratiquants, dont on affirme qu'ils sont possédés par les esprits tutélaires de la communauté. Le dénouement parfois heureux, parfois dramatique de ces possessions se présentera alors à l'assistance comme une indication de ce qui est mis en question par les esprits et qu'il faudra prendre en compte lors d'assemblées communes ou en effectuant les rituels appropriés.

\section{LE SYSTÈME DE TRANSMISSION ET L'ORDRE DES VALEURS}

On a vu que les techniques corporelles martiales s'inscrivent au sein d'un système de valeurs de référence de la communauté : islam, ancêtres, esprits protecteurs, travaux quotidiens, hiérarchie statutaire. Dans le même temps, elles permettent d'entrer en relation avec les esprits des ancêtres et les esprits de tigres. Leur transmission s'inscrit dans un ensemble rituel et s'effectue dans le cadre d'un système relationnel de type familial qui oriente le rapport aux conceptions de l'autorité et de la hiérarchie. Elle n'est donc pas dissociée de la transmission des droits et devoirs, des biens et statuts. Elle est profondément ancrée dans l'activité rituelle globale, la cosmologie et le système de classification sundanais. 
Ceci se traduit dans la relation au temps, où la pratique n'est pas détachée des autres activités et donc d'une perception globale du temps. Le secret qui entoure les généalogies de maîtres et la relation aux ancêtres corroborent le fait qu'il y a une valorisation de la profondeur généalogique. Spatialement, il y a une proximité entre le maitre et l'élève, ce qui permet de maintenir le contrôle sur le respect des règles et sur la préservation du secret qui entoure le système de transmission et les techniques. Par extension, cela signifie que le savoir-faire et la pratique sont valorisés et qu'ils ne sont pas séparés des savoirs théoriques qu'ils véhiculent.

On a décrit comment s'élabore la collaboration entre les élèves dans le cadre de la transmission, ce qui favorise le nivellement de l'accès à la connaissance. Dans ce cadre, la transmission fait partie de l'enseignement et la dimension relationnelle comme le vécu de l'action se trouvent valorisés par rapport à la finalité de l'action. L'échange relationnel tient une place prépondérante et semble être la clef du processus d'incorporation. Ici, transmettre c'est apprendre à se comporter, enseigner à vivre un certain type de relation dans un contexte où les situations sont reliées et font sens les unes en rapport avec les autres, participent d'une action vécue comme un tout (Grave, 2001).

Au niveau ultime, la créativité est encouragée comme preuve de maturité et de maitrise des techniques fondamentales du penca. La spécialisation possible d'un assistant et l'intégration de techniques nouvelles dans le système technique d'ensemble indiquent qu'il y a une participation de l'élève à la formation continue du maître et que les assistants sont valorisés en tant qu'intermédiaires dans le système d'apprentissage. Cependant, ces techniques intégrées le sont à un niveau subordonné du système technique d'ensemble, qui se maintient et qui incorpore ces éléments exogènes par rapport aux autres éléments du système et en relation avec des valeurs ordonnatrices. Par exemple, le rap- port à l'apprentissage est avant tout orienté par la notion de «jeu » et c'est en jouant au penca que l'on peut intégrer ou réintégrer des éléments exogènes ou des événements marquants dans l'ordre du système de valeurs, tout en leur accordant une place particulière au sein de celui-ci. Cet ordre est ainsi organisé comme un système ouvert où les parties sont ordonnées de façon hiérarchique et par niveaux. Ce système hiérarchisé, en intégrant les éléments exogènes selon une logique propre, joue donc le rôle d'un ensemble structurant bien plus que structurel.

\section{CONCLUSION}

En conclusion, on observe qu'il y a une relation d'interférence entre la technique et les valeurs sociales. La transmission du penca, qui s'insère dans une formation élargie du comportement et des aptitudes relationnelles indique que, pour les pratiquants, il n'y a pas de séparation de la théorie et de la pratique, du fait et de la valeur. Dans ce contexte, la technique semble profondément liée aux valeurs, et par conséquent ce qui appartient au domaine du psychomoteur est étroitement mêlé au ressenti et à la possession par les esprits. L'apprentissage de ce qui a trait au psychomoteur se fait par ricochets, de façon indirecte, comme un effet conjoint de la formation au ressenti ou de l'initiation rituelle qui met le pratiquant en contact avec les ancêtres et les esprits tutélaires, récipiendaires de la connaissance du penca.

Dans la dynamique de ce système, les nouvelles techniques introduites et les valeurs exogènes qu'elles véhiculent, se subordonnent à l'ordre des valeurs et ne le bouleversent pas en profondeur. Cependant, cela ne signifie pas qu'elles n'y apportent aucune modification; elles ne le font que graduellement et dans une logique propre à un système dans lequel la créativité et la valorisation de l'intégration d'éléments exogènes permettent de laisser place au changement. L'introduction d'éléments nouveaux 
est possible sans que le système de fond ne se désintègre. Et ce maintien atteste d'une dynamique structurante ouverte et intégratrice en même temps que d'un ancrage et d'une stabilité des valeurs de référence. Celles-ci apparaissent comme étroitement liées au système relationnel et c'est le maitre de penca et son système statutaire hiérarchique qui se portent garants de leur maintien.

Sur le plan du processus de transmission, au niveau élémentaire de la pratique, pour les enfants, une certaine directivité est mise en place et leur réflexivité est sollicitée. Ceci forme les capacités de l'intelligence motrice, tout en laissant une place importante à l'expérimentation et à l'autonomie. Les mouvements sont pratiqués à deux, les enfants viennent par petits groupes et ils n'ont une relation avec le maitre qui n'est qu'indirecte par le biais de leurs aînés. L'apprentissage du penca y prend la forme d'une socialisation par paliers, où les facultés motrices prennent progressivement le dessus sur la réflexivité. Aux niveaux élevés de pratique, c'est la formation au ressenti qui prend la place de la dimension réflexive. L'apprentissage des facultés liées à l'intelligence motrice se présente comme un effet contingent de cette formation et il s'inscrit dans le processus d'un savoir-être en société, d'un positionnement dans un ordre social hiérarchique.

\section{BIBLIOGRAPHIE}

Billeter, J.-F. (1984). Pensée occidentale et pensée chinoise : le regard et l'acte. In J.-C. Galey (Éd.), Différences, valeurs, hiérarchie. Textes offerts à Louis Dumont. Paris, EHESS, 25-51.

BruinesSen, M. V. (1999). Kitab Kuning: Pesantren dan Tarekat: Tradisi-Tradisi Islam di Indonesia. Penerbit Mizan, Bandung.

Dumont, L. (1992). Anthropologie, totalité et hiérarchie. In Philosophie et Anthropologie. Paris, Centre Georges Pompidou.

ENSERING, E. (1995). Banten in Times of Revolution. Archipel, 50, 131-161.

GRAVE, J. M. D. (2001). Initiation rituelle et arts martiaux. Trois écoles de kanuragan javanais. Paris, Archipel/L'Harmattan.

GraVE, J. M. D. (2007). L'initiation rituelle javanaise et ses modes de transmission. Techniques et culture, 48-49, $85-124$.

Kartodirdjo, S. (1995). Le leadership dans la révolte des paysans de Banten, 1888. Archipel, 50, 123-130.

MASAAKI, 0. (2004). Local politics in decentralized Indonesia: the governor general of Banten Province. IASS Newsletter, 34, 23.

Williams, M. C. (1990). Communism, religion, and revolt in Banten. Monographs in International Studies Southeast Asian Series, 86, Athens Ohio.

WiLSON, I. D. (2002). The politics of inner power: the practice of Pencak Silat in West Java. Thèse de doctorat non publiée, School of Asian studies. 